\title{
A similarity theory of locally homogeneous and isotropic turbulence generated by a Smagorinsky-type LES
}

\author{
By ANDREAS MUSCHINSKI \\ Institut für Meteorologie und Klimatologie der Universität Hannover, \\ 30419 Hannover, Germany
}

(Received 4 August 1995 and in revised form 13 May 1996)

A Kolmogorov-type similarity theory of locally homogeneous and isotropic turbulence generated by a Smagorinsky-type large-eddy simulation (LES) at very large LES Reynolds numbers is developed and discussed. The underlying concept is that the LES equations may be considered equations of motion of specific hypothetical fully turbulent non-Newtonian fluids, called 'LES fluids'. It is shown that the length scale $l_{S}=c_{S} \Delta$, which scales the magnitude of the variable viscosity in a Smagorinskytype LES, is the 'Smagorinsky-fluid' counterpart of Kolmogorov's dissipation length $\eta=v^{3 / 4} \varepsilon^{-1 / 4}$ for a Newtonian fluid where $v$ is the kinematic viscosity and $\varepsilon$ is the energy dissipation rate. While in a Newtonian fluid the viscosity is a material parameter and the length $\eta$ depends on $\varepsilon$, in a Smagorinsky fluid the length $l_{S}$ is a material parameter and the viscosity depends on $\varepsilon$. The Smagorinsky coefficient $c_{S}$ may be considered the reciprocal of a 'microstructure Knudsen number' of a Smagorinsky fluid. A combination of Lilly's (1967) cut-off model with two wellknown spectral models for dissipation-range turbulence (Heisenberg 1948; Pao 1965) leads to models for the LES-generated Kolmogorov coefficient $\alpha_{\text {LES }}$ as a function of $c_{S}$. Both models predict an intrinsic overestimation of $\alpha_{\text {LES }}$ for finite values of $c_{S}$. For $c_{S}=0.2$ Heisenberg's and Pao's models provide $\alpha_{\text {LES }}=1.74(16 \%$ overestimation $)$ and $\alpha_{\text {LES }}=2.14$ (43\% overestimation), respectively, if $\lim _{c_{S} \rightarrow \infty}\left(\alpha_{\mathrm{LES}}\right)=1.5$ is ad hoc assumed. The predicted overestimation becomes negligible beyond about $c_{S}=0.5$. The requirement $c_{S}>0.5$ is equivalent to $\Delta<2 l_{S}$. A similar requirement, $L<2 \eta$ where $L$ is the wire length of hot-wire anemometers, has been recommended by experimentalists. The value of $\lim _{\mathcal{C}_{S} \rightarrow \infty}\left(\alpha_{\text {LES }}\right)$ for a Smagorinsky-type LES at very large LES Reynolds numbers is not predicted by the models and remains unknown. Two critical values of $c_{S}$ are identified. The first critical $c_{S}$ is Lilly's (1967) value, which indicates the $c_{S}$ below which finite-difference-approximation errors become important; the second critical $c_{S}$ is the value beyond which the Reynolds number similarity is violated.

\section{Introduction}

The classical theory of homogeneous turbulence (Batchelor 1953) may be considered the backbone of the physics of fully developed turbulence. It is the present-day view (see, for example, Hunt, Phillips \& Williams 1991; Yaglom 1981, 1994) that the decisive breakthrough towards modern turbulence physics is to be attributed 
to Kolmogorov's (1941a,b) two classical papers. It is important to note, however, that Kolmogorov's similarity theory does not rely on the Navier-Stokes equations. Kolmogorov (1941a) did not even mention them. On the other hand, the NavierStokes equations are widely believed to be the first principles for the physics of fluid turbulence, also at high Reynolds numbers, and much work has been done to gain insight into Kolmogorov's turbulence laws from the viewpoint of the Navier-Stokes equations, see, for example, McComb (1990).

Since the advance of supercomputers, it has been possible to leave any turbulence theory completely aside and, instead, to straightforward numerically integrate the Navier-Stokes equations under the constraint of the external forcing. This approach is known as direct numerical simulation (DNS). See, for example, McComb (1990), Reynolds (1990) or Chen et al. (1993). DNS is free from any assumption on the statistical nature of developed turbulence and, to a certain extent, may be considered the numerical counterpart of a laboratory experiment. The DNS technique, however, is limited to moderate Reynolds numbers $R e$ since at very high $R e$ as encountered in the atmosphere or in the ocean the number of hydrodynamical degrees of freedom is far beyond present-day computer capacities.

A very efficient technique to reduce the numerical expense by many orders of magnitude is large-eddy simulation, abbreviated as LES (Smagorinsky 1963; Lilly 1967; Deardorff 1970). The technique's history and development have been compiled in a monograph edited by Galperin \& Orszag (1993). A critical review of the technique was given by Mason (1994).

LES relies on both the Navier-Stokes equations and on a reasonable model for the small-scale turbulence. In LES the Navier-Stokes equations and the other diagnostic and prognostic equations are used in a spatially filtered form. Lilly (1967) used a three-dimensional top-hat filter. Leonard (1974) generalized the filtering concept. Moeng \& Wyngaard (1988) compared 'empirical' spectra of the turbulent kinetic energy (TKE) generated in an LES with 'theoretical' TKE spectra that they obtained by applying the specific filter associated with their specific LES equations to Kolmogorov's inertial-range TKE spectrum. But the theoretical spectra did not compare well with the empirical LES spectra. They presumed that the discrepancy might be to be attributed to the fact that the theoretical spectra were obtained by explicitly filtering while the empirical LES TKE spectra were the result of a filtering operation that is "to some extent implicit" (Moeng \& Wyngaard 1988, p. 3577).

It is the purpose of this paper to give an elementary physical picture of this 'implicit' filtering. To a certain extent, we adopt the philosophy which was described by Mason (1994).

The paper is organized as follows. In $\S 2$, the difference between Navier-Stokes equations and LES equations is discussed. It is shown that LES equations may be considered equations of motion of specific hypothetical non-Newtonian turbulent fluids, called 'LES fluids'. Section 3 contains the essence of this paper. Somewhat in analogy to Kolmogorov's (1941a) similarity theory, a similarity theory of locally homogeneous and isotropic turbulence generated by a finite-difference Smagorinskytype LES is put forward. It is shown that the LES-generated Kolmogorov coefficient $\alpha_{\text {LES }}$ is sensitive to the Smagorinsky coefficient $c_{S}$ if $c_{S}$ is smaller than Lilly's (1967) value for $c_{S}$; on the other hand, $\alpha_{\text {LES }}$ is asymptotically universal for $c_{S}$ larger than Lilly's $c_{S}$. The similarity theory is generalized to account for turbulence generated by an anisotropic-grid LES. In $\S 4$, some implications of the similarity theory are discussed. A summary and conclusions are given in $\$ 5$. 


\section{The nature of the LES equations and the concept of LES fluids}

In this Section a physical interpretation of the nature of the LES equations will be given in some detail. It will be shown that the LES equations may be considered the equations of motion of specific hypothetical non-Newtonian turbulent fluids. These fluids will be called 'LES fluids'. The concept of LES fluids is useful to get a physical picture of the similarity theory of LES-generated turbulence that will be put forward in this paper.

\subsection{Navier-Stokes equations and LES equations; the concept of LES fluids}

DNS is a numerical integration of the Navier-Stokes equations. If a turbulent flow at a high Reynolds number $R e$ is to be modelled using DNS it must be guaranteed that the mesh width $\Delta$ of the numerical grid is smaller than the smallest curvature radii of the iso-surfaces of any physical quantity to be predicted by the DNS. It is generally accepted that this requirement is fulfilled if $\Delta$ is a few times smaller than Kolmogorov's (1941a) dissipation length

$$
\eta=\left(\frac{v^{3}}{\bar{\varepsilon}}\right)^{1 / 4}
$$

where $v$ is the molecular kinematic viscosity and $\bar{\varepsilon}$ the mean energy dissipation rate.

It is known that the ratio of the outer scale of the turbulence $L$ and the inner scale $\eta$ depends on the turbulent Reynolds number $R e$ :

$$
\frac{L}{\eta} \sim R e^{3 / 4} .
$$

Thus, DNS of a fully turbulent flow requires a number of grid points $N$ that increases dramatically as a function of $R e$ :

$$
N \sim\left(\frac{L}{\eta}\right)^{3} \sim R e^{9 / 4},
$$

see, e.g., Corrsin (1961).

Large-eddy simulation (LES) is a technique that allows numerical simulation of turbulent flows for arbitrarily large $R e$. In contrast to DNS, in LES not all turbulent structures are resolved but only the 'large' eddies, i.e. those having length scales larger than a certain length $l_{f}$. The 'inner inertial range', i.e. eddies having length scales between $\eta$ and $l_{f}$ is parameterized. The length $l_{f}$ is defined by the spatial filter that is applied to the Navier-Stokes equations in order to get a specific set of LES equations. Note that, like the Navier-Stokes equations, the LES equations are a priori partial differential equations. A numerical integration of the LES equations is called a LES.

The LES equations contain a term $\tau_{i j}$ that is physically interpreted as a Reynolds stress tensor that is variable in space and time. This tensor is to be parameterized in terms of the local instantaneous velocity field generated by the LES, and this is usually done on the basis of an eddy-viscosity hypothesis (Lilly 1967, p. 203; Leonard 1974, p. 240):

$$
\tau_{i j}=v_{\mathrm{LES}}\left(\frac{\partial u_{i}}{\partial x_{j}}+\frac{\partial u_{j}}{\partial x_{i}}\right) .
$$

Here, $v_{\text {LES }}$ is a kinematic viscosity which is not constant but varies temporally and spatially and depends on the local and instantaneous (a priori spatially filtered) velocity field $u_{i}$ generated by the LES. Now, in order to close the LES equations an 
additional equation is needed, namely the equation that allows the parameterization of $v_{\text {LES }}$ itself in terms of $u_{i}$.

A fluid in which the viscosity depends on the shear is a non-Newtonian fluid; therefore, the LES equations may be considered the equations of motion for a specific hypothetical non-Newtonian fluid. In the following, we will call such a hypothetical non-Newtonian fluid an 'LES fluid', which is physically specified by the equation for $v_{\text {LES }}$ or, more generally, by the parameterization of $\tau_{i j}$ in terms of the $u_{i}$.

The spatial filter that is inherent in the LES equations smears out the fluctuations that are considerably smaller than $l_{f}$. A physical interpretation is that the 'small' eddies are damped out by the eddy viscosity, which depends on (or defines) $l_{f}$ and which is usually several orders of magnitude larger than the molecular viscosity $v$ of the (Newtonian) fluid to be modelled. In other words $l_{f}$ is a property of the specific LES fluid while the molecular viscosity is a property of a Newtonian fluid. While the smallest eddies in a fully turbulent Newtonian fluid are on the order of the dissipation length $\eta$, the size of the smallest eddies in a fully turbulent (nonNewtonian) LES fluid is on the order of the filter length $l_{f}$. One might presume that the role that $\eta$ plays in Navier-Stokes turbulence is similar to that of $l_{f}$ in LES turbulence. In the next Sections we will show in more detail that, in full analogy to the dissipation length $\eta$ in a Newtonian fluid, $l_{f}$ is physically a dissipation length in an LES fluid.

The Navier-Stokes equations describe the energy dissipation of the turbulent kinetic energy due to the random motion of the molecules. The molecular motion itself, however, is not explicitly described but is parameterized. In other words the Navier-Stokes equations do not know anything about the existence of individual molecules; in a Navier-Stokes fluid (a fluid that is described by the Navier-Stokes equations), there are a priori no structures at length scales considerably smaller than $\eta$. Even if it were possible to carry out a DNS with a grid spacing $\Delta$ smaller than the size of the molecules one could not expect that the DNS would provide insight into the existence of individual molecules. Correspondingly, the LES equations describe the energy dissipation of the large eddies due to the effects of the eddies smaller than $l_{f}$. The motion of the small eddies itself, however, is not explicitly described but is parameterized. In other words the LES equations do not know anything about the existence of individual eddies smaller than $l_{f}$, i.e. of eddies within the inner inertial range; in an LES fluid, there are a priori no structures at length scales considerably smaller than $l_{f}$. Even if a LES were carried out with $\Delta$ smaller than $\eta$ one could not expect that the LES would resolve the eddies smaller than $l_{f}$ if $l_{f}$ is several orders of magnitude larger than $\eta$, which is usually the case.

It is important to note that up to now we have considered partial differential equations (or finite-difference equations with an arbitrarily small $\Delta$ ): on the one hand the Navier-Stokes equations and on the other hand the LES equations. As stated above, in DNS $\Delta$ must be chosen equal to or smaller than a fraction of $\eta$ since the size of the smallest nonlinear structures in a turbulent Newtonian fluid is on the order of $\eta$. Correspondingly, in LES $\Delta$ must be chosen smaller than a critical length that is defined by $l_{f}$ (Mason 1994, p. 5).

The LES technique was developed to minimize the computational expense of the simulation of turbulent flows with very high Reynolds numbers. Thus, there is generally a need to chose $\Delta$ as large as possible, and there is a need to know the critical $\Delta$ as precisely as possible. Note, however, that the need to maximize $\Delta$ is simply a consequence of limited computer resources. Thus, the grid spacing is maximized for technical reasons but not for physical reasons. 
Obviously, the value of $\Delta$ must have an influence on the LES results if $\Delta$ is close to or even larger than the critical $\Delta$, which depends on $l_{f}$ since $l_{f}$ defines the length scales of the smallest structures in the LES-generated velocity field. On the other hand, however, it is to be expected that for a given $l_{f}$ the LES results will be insensitive to $\Delta$ if it is chosen considerably smaller than its critical value.

In summary, we point out that it is very important to carefully distinguish between physically resolved length scales and numerically resolved length scales, and it is to be realized that the spatial filter defined by the model for $\tau_{i j}$ has a priori nothing to do with the numerical grid that is used for the numerical integration of the LES equations.

\subsection{A specific LES fluid: the Smagorinsky fluid}

Following Smagorinsky (1963), Lilly (1967) suggested an eddy viscosity $v_{\mathrm{LES}}$ that is proportional to the deformation tensor amplitude:

$$
v_{\mathrm{LES}}=l_{S}^{2} S
$$

where

$$
S=\left[\frac{1}{2}\left(\frac{\partial u_{i}}{\partial x_{j}}+\frac{\partial u_{j}}{\partial x_{i}}\right)^{2}\right]^{1 / 2},
$$

compare, for example, Leonard (1974), Schmidt \& Schumann (1989), and Mason \& Brown (1994). We call an LES fluid defined by (2.5) and (2.6) a 'Smagorinsky fluid' and the length $l_{S}$ the 'Smagorinsky length'. Usually, but not necessarily, $l_{S}$ is stated in units of a grid spacing $\Delta$,

$$
l_{S}=c_{S} \Delta
$$

where the numerical coefficient $c_{S}$ is known as the 'Smagorinsky coefficient' (e.g. Schmidt \& Schumann 1989, p. 556) or the 'Smagorinsky constant' (e.g. Germano et al. 1991). It is to be emphasized, however, that there is no need to introduce a finite grid spacing $\Delta$ at this point. The coefficient $c_{S}$ has no physical relevance as long as the LES equations, which are a priori partial differential equations, are not replaced with their finite-difference counterparts.

Obviously, $l_{S}$ defines the magnitude of $v_{\text {LES }}$ and simultaneously the magnitude of $\tau_{i j}$. Since, in turn, the parameterization of $\tau_{i j}$ in terms of $u_{i}$ defines the effective spatial filter associated with the LES equations the filter length $l_{f}$ is defined by $l_{S}$. In the next Section, we will show that the Smagorinsky length $l_{S}$ is the Smagorinsky-fluid counterpart of the Navier-Stokes-fluid dissipation length $\eta$.

\section{Spectral analysis of homogeneous and isotropic LES-generated turbulence}

\subsection{The effective spatial filter}

There are two different interpretations of the nature of the spatial filter inherent in the LES equations. The traditional approach (Lilly 1967; Leonard 1974) is as follows. The Navier-Stokes equations are spatially filtered. The filter is explicitly defined and a priori known. The result are equations of motion, the form of which is "precisely that of the Navier-Stokes equations with $\tau_{i j}$ replacing the viscous term" (Mason 1994, p. 3). Thus, the Reynolds stress tensor $\tau_{i j}$ contains the full information concerning the 
spatial filter. If $\tau_{i j}$ is properly parameterized the LES will provide the TKE spectrum

$$
F(k)=\alpha \bar{\varepsilon}^{2 / 3} k^{-5 / 3} A^{2}(k),
$$

where $A(k)$ is the transfer function which is the Fourier transform of the spatial filter function (Leonard 1974, equations 4.23 and 4.24; see also Moeng \& Wyngaard 1988, pp. 3574ff.). Since the filter is a priori known the transfer function and the LES-generated TKE spectrum are also a priori known. So far this is the traditional philosophy.

An alternative interpretation has been given by Mason and his co-workers (Mason \& Callen 1986; Mason \& Brown 1994; Mason 1994). The pivotal issue is that in an LES $\tau_{i j}$ is to be parameterized in terms of the filtered field variables, but the relation between $\tau_{i j}$ and the filtered variables is not exactly known. The Smagorinsky-type parameterization, for example, is reasonable but nevertheless ad hoc. Mason (1994, p. 4): "Since the Smagorinsky model only involves a single scalar variable $l_{0}$ [the Smagorinsky-length $l_{S}$ in the present paper] it is immediately apparent that, whatever the approach, only the characteristic scale of a filter can be represented. A key, but as yet unanswered, question is: what particular shape of filter operation does the Smagorinsky model correspond to?"

This is the central question that the present paper deals with. We will not give a conclusive answer but we will see that the physical nature of this effective 'Smagorinsky-filter' is similar to the 'diffuse cutoff' (Moeng \& Wyngaard 1988, p. 3578) of the inertial-range TKE spectrum of Navier-Stokes turbulence at wavenumbers on the order of $\eta^{-1}$. In the next subsection we interpret turbulence generated by a Smagorinsky-type LES as turbulence in a hypothetical Smagorinsky fluid and get some insight into the LES-generated ('resolved-scale') TKE spectrum by making use of a Kolmogorov-type dimensional analysis.

\subsection{Dimensional analysis of homogeneous and isotropic turbulence in a Smagorinsky fuid}

Assuming $L \gg l_{S}$ we postulate three similarity hypotheses for statistically isotropic and homogeneous turbulence generated by a cubic-grid LES that relies on a Smagorinskytype parameterization of the Reynolds stress tensor:

First similarity hypothesis: $F(k)$ is determined by $\bar{\varepsilon}, l_{S}$ and $A$.

Second similarity hypothesis: At wavenumbers $k$ considerably smaller than $l_{S}^{-1}, F(k)$ is determined by $\bar{\varepsilon}$ and $\Delta$ but does not depend on $l_{S}$.

Third similarity hypothesis: $F(k)$ is determined by $\bar{\varepsilon}$ and $l_{S}$ but does not depend on $\Delta$ if $\Delta$ is considerably smaller than $l_{S}$.

The first two hypotheses are similar to Kolmogorov's (1941a) two hypotheses. The third hypothesis, however, is postulated because the LES equations are a priori partial differential equations and because it is to be expected that the numerical solution of these equations becomes asymptotically independent of $\Delta$ if $\Delta$ is chosen considerably smaller than a certain critical value (Mason 1994, pp. 7ff.).

Defining the two dimensionless parameters

$$
\Pi_{1}=k l_{s}
$$

and

$$
\Pi_{2}=\frac{\Delta}{l_{S}}=\frac{1}{c_{S}},
$$


and making use of the $\Pi$-theorem (see, e.g., Görtler 1975 and the references cited by him) we find that in the general case the LES-generated TKE spectrum may be written

$$
F(k)=\bar{\varepsilon}^{2 \cdot / 3} k^{-5 / 3} G\left(\Pi_{1}, \Pi_{2}\right),
$$

where $G$ is a dimensionless function of two dimensionless variables. The inertialrange limit of $G\left(\Pi_{1}, \Pi_{2}\right)$ is $G\left(0, \Pi_{2}\right)$ and may be considered the LES counterpart of the Kolmogorov constant $\alpha$ :

$$
\alpha_{\mathrm{LES}}\left(c_{S}\right)=G\left(0, \Pi_{2}\right)
$$

Note that in the general case the LES-generated Kolmogorov coefficient $\alpha_{\text {LES }}$ is a function of $c_{S}$. Introducing

$$
f_{\mathrm{LES}}\left(k l_{S}, c_{S}\right)=\frac{G\left(\Pi_{1}, \Pi_{2}\right)}{G\left(0, \Pi_{2}\right)}
$$

provides

$$
F(k)=\alpha_{\mathrm{LES}}\left(c_{S}\right) \bar{\varepsilon}^{2 / 3} k^{-5 / 3} f_{\mathrm{LES}}\left(k l_{S}, c_{S}\right)
$$

where

$$
\lim _{x \rightarrow 0} f_{L E S}\left(x, c_{S}\right)=1
$$

for any value of $c_{S}$.

3.3. Lilly's (1967) assumptions considered from the viewpoint of the similarity theory Lilly's (1967) well-known relationship between the Smagorinsky coefficient and the Kolmogorov constant $\alpha$ can be rederived from the general LES TKE spectrum, (3.7), as follows. Lilly (1967) assumed

$$
f_{\text {LES }}\left(k c_{S} \Delta, c_{S}\right)=\left\{\begin{array}{lll}
1 & \text { for } & k \leqslant \pi / \Delta \\
0 & \text { for } & k>\pi / \Delta
\end{array}\right.
$$

and

$$
\alpha_{\mathrm{LES}}\left(c_{S}\right)=\alpha,
$$

where $\alpha$ is the quasi-universal Kolmogorov constant which is empirically known from real-world turbulence. Muschinski \& Roth (1993) suggested a local effective cutoff wavenumber $\pi /(2 z)$ (where $z$ is the distance from the surface) for surface-layer turbulence. Schumann (1994) pointed out that this cut-off wavenumber is closely related to Lilly's (1967) cut-off wavenumber $\pi / \Delta$ for LES-generated turbulence.

Making use of approximations for the ensemble averages $\bar{\varepsilon}$ and $\overline{\nu_{\text {LES }}}$ in homogeneous LES turbulence,

$$
\bar{\varepsilon}=2 \overline{v_{\mathrm{LFS}}} \int_{0}^{\infty} F(k) k^{2} \mathrm{~d} k
$$

and

$$
\overline{v_{\mathrm{LES}}}=\left(c_{S} \Delta\right)^{2}\left(2 \int_{0}^{\infty} F(k) k^{2} \mathrm{~d} k\right)^{1 / 2}
$$


one obtains Lilly's (1967) result

$$
c_{S}=\frac{1}{\pi}\left(\frac{3 \alpha}{2}\right)^{3 / 4} .
$$

3.4. The nature of the Smagorinsky length

The Smagorinsky length $l_{S}$ defines the magnitude of the variable kinematic viscosity of a fully turbulent Smagorinsky fluid, see (2.5). In analogy to Kolmogorov's (1941a) dissipation length, (2.1), we define a dissipation length for a LES fluid:

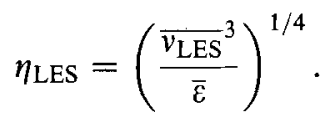

Using (3.11) and (3.12) we obtain

$$
\eta_{\mathrm{LES}}=c_{S} \Delta=l_{S} .
$$

Thus, we have shown that the Smagorinsky-length is the dissipation length of the Smagorinsky-fluid, and we can rewrite (3.7):

$$
F(k)=\alpha_{\text {LES }}\left(c_{S}\right) \bar{\varepsilon}^{2 / 3} k^{-5 / 3} f_{\text {LES }}\left(k \eta_{\text {LES }}, c_{S}\right) .
$$

3.5. Comparison with Kolmogorov's (1941) similarity theory

According to Kolmogorov's (1941a) similarity theory, the three-dimensional TKE spectrum in locally homogeneous and isotropic fully developed turbulence is uniquely determined by the mean energy dissipation rate $\bar{\varepsilon}$ and the molecular kinematic viscosity $v$ :

$$
F(k)=\alpha \bar{\varepsilon}^{2 / 3} k^{-5 / 3} f(k \eta),
$$

where

$$
\eta=\left(\frac{v^{3}}{\bar{\varepsilon}}\right)^{1 / 4}
$$

and

$$
\lim _{x \rightarrow 0} f(x)=1 .
$$

In the early 1940s, it was pointed out by Landau that the spatial distribution of $\varepsilon$ should be taken into account in a reliable similarity theory for turbulence at high Reynolds numbers. This was done by Kolmogorov (1962), but the influence of the small-scale intermittency on the shape of the TKE spectrum has proved to be small. It is negligible in many applications.

According to the third similarity hypothesis postulated above we expect for large Smagorinsky coefficients:

$$
F(k)=\left(\lim _{c_{S} \rightarrow \infty} \alpha_{\mathrm{LES}}\left(c_{S}\right)\right) \bar{\varepsilon}^{2 / 3} k^{-5 / 3}\left(\lim _{c_{S} \rightarrow \infty} f_{\mathrm{LES}}\left(k \eta_{\mathrm{LES}}, c_{S}\right)\right) .
$$

It is tempting to presume

$$
\lim _{c_{S} \rightarrow \infty} \alpha_{\mathrm{LES}}\left(c_{S}\right) \approx \alpha
$$

and

$$
\lim _{c_{S} \rightarrow \infty} f_{\mathrm{LES}}\left(x, c_{S}\right) \approx f(x)
$$


where $\alpha$ is the quasi-universal Kolmogorov constant and $f(x)$ the quasi-universal 'damping function' for Navier-Stokes turbulence. In other words: one might presume that Kolmogorov's (1941a) similarity theory not only holds for Navier-Stokes turbulence but also for turbulence generated by a Smagorinsky-type LES if $c_{S}$ is sufficiently large.

This presumption appears to be justified if the details of the spatial and temporal distribution of $v_{\text {LES }}$ in a fully turbulent Smagorinsky fluid may be ignored and if its mean value is the important parameter that characterizes the TKE spectrum. Such a ' $v_{\mathrm{LES}}$-homogeneity assumption' is encouraged by the success of the $\varepsilon$-homogeneity assumption that Kolmogorov's (1941a) theory relies on. Moreover, the numerical experiments carried out by Bardina, Ferziger \& Reynolds (1983) and by Mason \& Brown (1994) may also be seen as an empirical verification of the unimportance of the details of the distribution of $v_{L E S}$ in the case of homogeneous turbulence: "In the flow interior, it seems that only the mean value of the eddy-viscosity is at issue" (Mason \& Brown 1994, p. 134).

\subsection{LES dissipation spectra}

The normalized dissipation spectrum is given by

$$
g_{\mathrm{LES}}\left(x, c_{S}\right)=x^{1 / 3} f_{\mathrm{LES}}\left(x, c_{S}\right)
$$

where

$$
x=k \eta_{\text {LES }}
$$

is the dimensionless wavenumber. Figure 1 shows three models for $g_{\text {LES }}\left(x, c_{S}\right)$. Two of the three curves correspond with the semi-empirical damping functions after Heisenberg (1948),

$$
f_{H}(x)=\left(1+\left(\frac{3 \alpha}{2}\right)^{3} x^{4}\right)^{-4 / 3}
$$

and Pao (1965),

$$
f_{P}(x)=\exp \left(-\frac{3 x}{2} x^{4 / 3}\right),
$$

respectively. We have assumed the standard value $\alpha=1.5$. Although the discussion about the definitive asymptotic form of $f(x)$ for large $x$ has not been settled (see, for example, Schumann 1994 and Saddoughi \& Veerevalli 1994), the models by Heisenberg (1948) and Pao (1965) are used here since they fulfil the accuracy requirements that are appropriate in the present context.

The third curve in figure 1 is the dissipation spectrum that follows from Lilly's (1967) model, see (3.13) and (3.9):

$$
g_{L}(x)=\left\{\begin{array}{ll}
x^{1 / 3} & \text { for } x \leqslant x_{s} \\
0 & \text { for } x>x_{s}
\end{array},\right.
$$

where

$$
x_{s}=\left(\frac{2}{3 \alpha}\right)^{3 / 4}
$$

is the dimensionless cut-off wavenumber according to Lilly (1967). In figure $1, \alpha=1.5$ has been assumed for Lilly's dissipation spectrum also. 


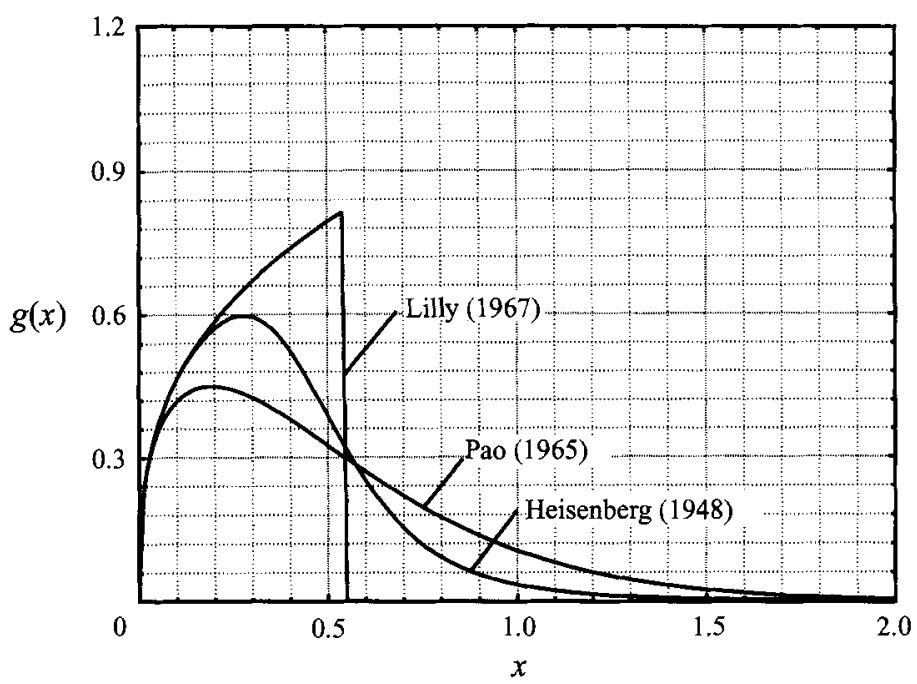

FIGURE 1. Models for the dimensionless dissipation spectrum for locally homogeneous and isotropic turbulence generated by a Smagorinsky-type LES. The three curves depict the dissipation spectra according to the models by Heisenberg (1948), Lilly (1967), and Pao (1965), respectively.

\subsection{The LES-generated Kolmogorov coefficient as a function of the Smagorinsky coefficient}

Now we derive the LES-generated Kolmogorov coefficient as a function of the Smagorinsky coefficient. Combining (3.11) and (3.12) yields

$$
\bar{\varepsilon}=\left(c_{S} \Delta\right)^{2}\left(2 \int_{0}^{\infty} F(k) k^{2} \mathrm{~d} k\right)^{3 / 2} .
$$

Inserting (3.7) and resolving for $\alpha_{\text {LES }}$ leads to

$$
\alpha_{\mathrm{LES}}\left(c_{S}\right)=\frac{1}{2 \int_{0}^{\infty} x^{1 / 3} f_{\mathrm{LES}}\left(x, c_{S}\right) \mathrm{d} x}
$$

where

$$
x=k l_{S}=k c_{S} \Delta .
$$

A simple model for $f_{\mathrm{LES}}\left(x, c_{S}\right)$ is the large- $c_{S} \operatorname{limit}, \lim _{c_{S} \rightarrow \infty} f_{\mathrm{LES}}\left(x, c_{S}\right)$, cut-off at

$$
x_{s}=k_{s} c_{S} \Delta=\pi c_{S} .
$$

Such a cut-off has already been suggested by Lilly (1967) but simultaneously he made use of the crude assumption $f(x)=1$. Assuming Kolmogorov's (1941a) universal damping function, $f(x)$, as the large- $c_{S}$ limit of $f_{\mathrm{LES}}\left(x, c_{S}\right)$, see (3.22), we obtain

$$
\alpha_{\mathrm{LES}}\left(c_{S}\right)=\frac{1}{2 \int_{0}^{\pi c_{S}} x^{1 / 3} f(x) \mathrm{d} x} .
$$

It is easy to verify Lilly's $\alpha-c_{s}$-relationship, (3.13), for $f(x)=1$.

As two more precise models for $f(x)$, which take the decoupling of $\alpha_{\mathrm{LES}}$ from $c_{S}$ for large $c_{S}$ into account (which Lilly's model does not), we suggest the above-mentioned 
models by Heisenberg (1948) and Pao (1965). Inserting Heisenberg's model, (3.25), into (3.33) reveals a model for $\alpha_{\mathrm{LES}}\left(c_{S}\right)$ in closed form. After solving the integral and after some elementary manipulations we obtain

$$
\alpha_{\mathrm{LES}}\left(c_{S}\right)=\alpha\left(1+\left(\frac{2}{3 \alpha}\right)^{3}\left(\pi c_{S}\right)^{-4}\right)^{1 / 3}
$$

This result and the alternative model for $\alpha_{\mathrm{LES}}\left(c_{S}\right)$ which relies on Pao's (1965) model will be discussed in $\$ 4$.

\subsection{Dimensional analysis of homogeneous and isotropic turbulence generated with an anisotropic-grid LES}

Consider a finite-difference Smagorinsky-type LES with an anisotropic grid. Let $\Delta_{1}$, $\Delta_{2}$ and $\Delta_{3}$ be the three grid spacings, where

$$
\Delta_{1} \leqslant \Delta_{2} \leqslant \Delta_{3}
$$

We modify the similarity theory described above in order to account for anisotropic grids. Assuming again $L \gg l_{S}$ we postulate:

First similarity hypothesis: $F(k)$ is determined by $\bar{\varepsilon}, l_{S}, \Delta_{1}, \Delta_{2}$, and $\Delta_{3}$.

Second similarity hypothesis: At wavenumbers $k$ considerably smaller than $l_{S}^{-1}, F(k)$ is determined by $\bar{\varepsilon}, \Delta_{1}, \Delta_{2}$, and $\Delta_{3}$ but does not depend on $l_{S}$.

Third similarity hypothesis: $F(k)$ is determined by $\bar{\varepsilon}$ and $l_{S}$ but does not depend on $\Delta_{1}, \Delta_{2}$, and $\Delta_{3}$ if $\Delta_{1}, \Delta_{2}$, and $\Delta_{3}$ are considerably smaller than $l_{S}$.

We define four dimensionless variables:

$$
\begin{gathered}
\Pi_{1}=k l_{S}, \\
\Pi_{2}=\frac{1}{c_{S}}=\frac{\Delta_{D}}{l_{S}}, \\
\Pi_{3}=a_{1}=\frac{\Delta_{1}}{\Delta_{3}},
\end{gathered}
$$

and

$$
\Pi_{4}=a_{2}=\frac{\Delta_{2}}{\Delta_{3}}
$$

where

$$
\Delta_{D}=\left(\Delta_{1} \Delta_{2} \Delta_{3}\right)^{1 / 3}
$$

is a measure for the grid spacing as used by Deardorff (1970), and where $a_{1}$ and $a_{2}$ are 'aspect ratios' that quantify the anisotropy of the grid (Scotti, Meneveau \& Lilly 1993). The $\Pi$-theorem leads to

$$
F(k)=\bar{\varepsilon}^{2 / 3} k^{-5 / 3} G_{a}\left(\Pi_{1}, \Pi_{2}, \Pi_{3}, \Pi_{4}\right)
$$

where $G_{a}$ is a dimensionless function of four dimensionless variables. The suffix $a$ stands for anisotropic.

We normalize $G_{a}\left(\Pi_{1}, \Pi_{2}, \Pi_{3}, \Pi_{4}\right)$ with the inertial-range limit, $G_{a}\left(0, \Pi_{2}, \Pi_{3}, \Pi_{4}\right)$, and get

$$
F(k)=\alpha_{\mathrm{LES}}\left(c_{S}\right) \beta_{a}\left(c_{S}, a_{1}, a_{2}\right) \bar{\varepsilon}^{2 / 3} k^{-5 / 3} f_{a}\left(k l_{S}, c_{S}, a_{1}, a_{2}\right)
$$


where

$$
f_{a}\left(k l_{S}, c_{S}, a_{1}, a_{2}\right)=\frac{G_{a}\left(\Pi_{1}, \Pi_{2}, \Pi_{3}, \Pi_{4}\right)}{G_{a}\left(0, \Pi_{2}, \Pi_{3}, \Pi_{4}\right)} .
$$

$G_{a}\left(0, \Pi_{2}, \Pi_{3}, \Pi_{4}\right)$ is the LES-generated Kolmogorov coefficient in the case of an anisotropic grid, and it is appropriate to express it in terms of the LES-generated Kolmogorov coefficient in the case of an isotropic grid and an 'anisotropy factor' $\beta_{a}$ which depends in the general case on $c_{S}, a_{1}$ and $a_{2}$ :

$$
\alpha_{\mathrm{LES}}\left(c_{S}\right) \beta_{a}\left(c_{S}, a_{1}, a_{2}\right)=G_{a}\left(0, \Pi_{2}, \Pi_{3}, \Pi_{4}\right) .
$$

In the case of an isotropic grid we have

$$
\beta_{a}\left(c_{S}, 1,1\right)=1 .
$$

Scotti et al. (1993) generalized Lilly's (1967) analysis for an anisotropic grid, assuming a three-dimensional anisotropic cut-off filter defined by three different cut-off wavenumbers, corresponding with the three different grid spacings. Moreover, they assumed

$$
\alpha_{\mathrm{LES}}\left(c_{S}\right) \beta_{a}\left(c_{S}, a_{1}, a_{2}\right)=\alpha
$$

and obtained $c_{S}$ as a function of the empirical Kolmogorov constant $\alpha$ and of the grid aspect ratios $a_{1}$ and $a_{2}$.

Finally we consider the case that $\Delta_{1}, \Delta_{2}$, and $\Delta_{3}$ are considerably smaller than $l_{S}$. Then, according to the third similarity hypothesis, $\alpha_{\mathrm{LES}}\left(c_{S}\right)$ is equal to the isotropic large- $c_{S}$ limit, and $\beta_{a}\left(c_{S}, a_{1}, a_{2}\right)=1$ even for an anisotropic-grid LES.

It seems possible that dimensional analysis can lead to further insight into the asymptotic behaviour of the anisotropy function $\beta_{a}\left(c_{S}, a_{1}, a_{2}\right)$ for various limits of $c_{S}$, $a_{1}$, and $a_{2}$. I believe in this case, instead of making use of $c_{S}, a_{1}$, and $a_{2}$, it would be more appropriate to introduce three different Smagorinsky coefficients $c_{S 1}=l_{S} / \Delta_{1}$, $c_{S 2}=l_{S} / \Delta_{2}$, and $c_{S 3}=l_{S} / \Delta_{3}$. As one of the reviewers pointed out, it would be of interest to consider the case that one (two) of the three Smagorinsky coefficients is (are) small and two (one) are (is) large. A detailed discussion of this problem, however, is beyond the scope of this paper.

\section{Discussion}

\subsection{The relevance of the Smagorinsky coefficient for the LES-generated TKE} spectrum and the LES-generated Kolmogorov coefficient

Figure 1 shows clearly the difference between LES with a small $c_{S}$ and a LES with a large $c_{S}$. The dissipation spectra $g_{H}(x)$ and $g_{P}(x)$ have maxima at wavenumbers $x$ about half the cut-off wavenumber $x_{s}$ suggested by Lilly (1967), indicating insensitivity of the TKE budget of the LES-generated turbulence if $x_{s}$ is chosen considerably larger than Lilly's $x_{s}$. The maximum of Lilly's crude cut-off dissipation spectrum, $g_{L}(x)$, however, is at $x_{s}$, implying a priori a coupling between the TKE budget and both the magnitude of the cut-off wavenumber and of the grid spacing $\Delta$.

Figure 2 shows several models for $\alpha_{\text {LES }}\left(c_{S}\right)$. The solid lines represent $\alpha_{\text {LES }}\left(c_{S}\right)$ obtained from inserting $f_{L}(x), f_{H}(x)$, and $f_{P}(x)$, respectively, into Eq. (3.33). The dashed line marks the value of $\lim _{c_{S} \rightarrow \infty}\left(\alpha_{\text {LES }}\right)$, which has ad hoc been assumed to be 1.5. Since Lilly's (1967) model assumes a cut-off of the spectrum at the largest wavenumber resolvable by the grid, it is a priori clear that it fails for larger $c_{S}$, i.e. in the case of a decoupling of the LES turbulence from the numerics. Note that 


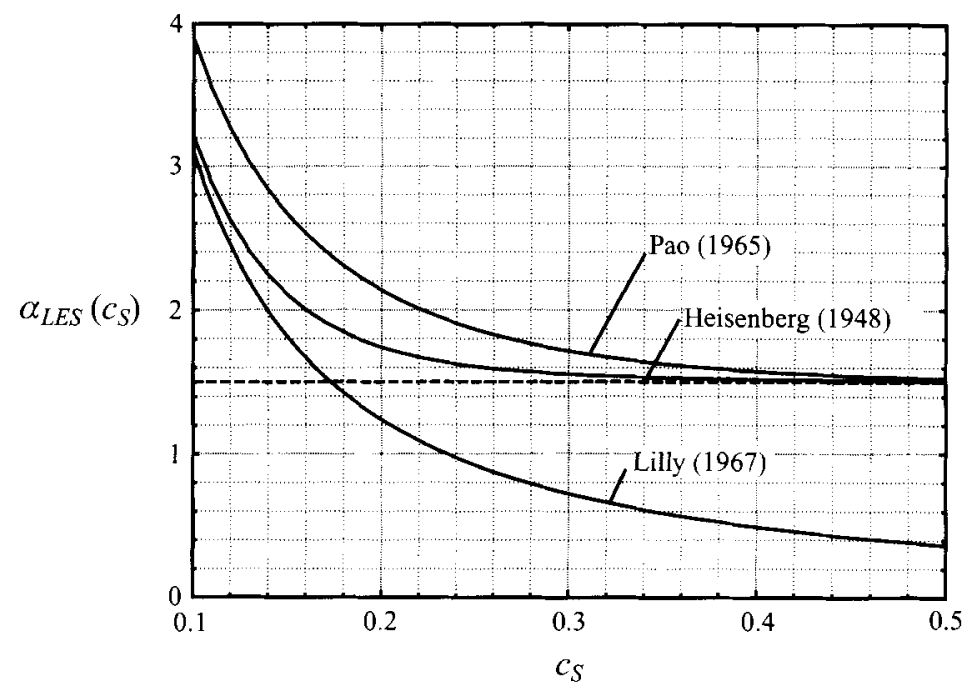

Figure 2. Models for the Kolmogorov coefficient $\alpha_{\text {LES }}$ generated by a Smagorinsky-type LES as a function of the Smagorinsky coefficient $c_{S}$. The three solid curves show $\alpha_{\text {LES }}\left(c_{S}\right)$ according to Lilly (1967) and two models for $\alpha_{\text {LES }}\left(c_{S}\right)$ that rely on the spectral models by Heisenberg (1948) and Pao (1965), respectively. For the asymptotic value, $\lim _{C S \rightarrow \infty} x_{L E S}\left(c_{S}\right) 1.5$ has been assumed (dashed line).

the Lilly asymptote and the (constant) large- $c_{S}$ asymptote intersect exactly at Lilly's (1967) value for $c_{S}$. Thus, Lilly's (1967) $c_{S}$ may be considered a critical $c_{S}$.

Both Pao's (1965) and Heisenberg's (1948) models also imply a systematic overestimation of $\alpha_{\text {LES }}$ for $c_{S}$ larger than 0.2 if the energy at the smallest grid scales is not artificially removed. Pao's (1965) model leads to a larger predicted overestimation than Heisenberg's (1948) because the 'diffuse cut-off' of the TKE spectrum according to Pao's model is more diffuse than that according to Heisenberg's model, see figure 1. For $\lim _{c_{S} \rightarrow \infty}\left(\alpha_{\mathrm{LES}}\right)=1.5$ and $c_{S}=0.2$ we obtain $\alpha_{\mathrm{LES}}=1.74$ (16\% overestimation) from Heisenberg's model and $\alpha_{\text {LES }}=2.14$ (43\% overestimation) from Pao's model. A possible intrinsic overestimation of LES-generated Kolmogorov coefficients has been reported by Chasnov (1991): “... it appears that the value of $K o\left[\alpha_{\mathrm{LES}}\right.$ in the present paper] obtained from numerical simulations is approximately $30 \%$ higher than that obtained in high Reynolds number atmospheric experiments. The origin of this discrepancy remains to be understood." Chasnov (1991) tested a possible cut-off effect by comparing a $64^{3}$ run with a $128^{3}$ run and obtained an even slightly larger $\alpha_{\text {LES }}$ for the $128^{3}$ run than for the $64^{3}$ run, which seems to exclude that the overestimation is to be attributed to a truncation effect. On the other hand, the physics of Chasnov's spectral wavenumber-space LES, which is based on a spectral subfilter closure, is different from the physics of a basic finite-difference Smagorinsky-type LES, which is carried out in physical space. Hence, the reliability of a direct comparison between Chasnov's results and results expected for the basic Smagorinsky-type LES, might be doubtful.

In most LES applications, values for $c_{S}$ between 0.1 and 0.2 have been used. These values are fairly close to Lilly's (1967) value, which marks the intermediate range between the small- $c_{S}$ asymptote and the large- $c_{S}$ asymptote. Using a $c_{S}$ in the intermediate range has three practical advantages: first, the LES-generated Kolmogorov coefficient is not very sensitive to $c_{S}$; second, the LES-generated Kolmogorov coeffi- 
cient is expected to be not too far from the quasi-universal Kolmogorov coefficient $\alpha$; third, the numerical expense of the LES is minimized.

For large values of $c_{S}$ the length scale defined by the model for $\tau_{i j}$ (and not the grid spacing) determines the width of the effective spatial filter. For small values of $c_{S}$, however, the effective spatial filter is determined by the numerical grid. In a small- $c_{S}$ LES, the grid spacing $\Delta$ plays the role of the transport length that determines the magnitude of the effective subfilter viscosity. In the intermediate range, i.e. at Smagorinsky coefficients close to Lilly's (1967) value, obviously both the numerical grid and the $\tau_{i j}$ closure determine the effective spatial filter. Thus, in an intermediate$c_{S}$ LES the nature of the turbulent motions at the smallest scales is ambiguous. The physical properties are defined at distinct points, namely at the grid points. One might speculate that for small and intermediate values of $c_{S}$ the dynamics of the velocity fluctuations at scales comparable with the grid spacing is somewhat similar to the microphysical dynamics of a solid at the smallest scales.

The grid spacing may be considered the LES-fluid counterpart of the mean free path in a gaseous Navier-Stokes fluid. Tennekes \& Lumley (1972, p. 23) called the ratio between the mean free path and the dissipation length,

$$
K n_{m}=\frac{\lambda}{\eta},
$$

a "microstructure Knudsen number". It is generally expected that turbulence can be properly described within the framework of continuum mechanics as long as $K n_{m}$ is smaller than 1. The LES-fluid counterpart of $K n_{m}$ is

$$
K n_{m}=\frac{\Delta}{\eta_{\mathrm{LES}}}=\frac{\Delta}{l_{S}}=\frac{1}{c_{S}} .
$$

Thus, the Smagorinsky coefficient is physically the reciprocal of a microstructure Knudsen number (or a 'grid Knudsen number'). Mason (1994, p. 8), however, considers $c_{S}$ the reciprocal of the square root of a "mesh Reynolds number".

Figure 2 indicates that the overestimation becomes negligible for $c_{S}$ larger than about 0.5. This is equivalent to the requirement $\Delta<2 l_{s}$. Experimentalists (see Kutznetsov, Praskovsky \& Sabelnikov 1992, p. 602) have recommended using a wire length $L$ (which defines the spatial resolution in hot-wire anemometry) smaller than $2 \eta$ for investigations of fully developed turbulence. Since $l_{S}$ is the Smagorinskyfluid counterpart of $\eta$ the requirement $c_{S}<0.5$ is the Smagorinsky-fluid counterpart of the requirement $L<2 \eta$. This analogy may be seen as a further indication for the reasonableness of the presumption that there is a similarity between the 'diffuse cut-offs' in Smagorinsky-fluid turbulence and in real-world turbulence.

\subsection{Subgrid scales, subfilter scales, and resolved scales}

It is worthwhile to look more closely at the different terminologies used by the different authors. Most researchers in the LES community (see, e.g., Deardorff 1980; Moeng \& Wyngaard 1988; Schmidt \& Schumann 1989; Kaltenbach, Gerz \& Schumann 1994) use the term 'subgrid scales'. Mason and his co-workers (see, e.g., Mason 1994; Mason \& Brown 1994), however, prefer the term 'subfilter scales'. A third notion is 'resolved scales'. What is the difference between these terminologies? Here I suggest a definition. 
Subgrid scales are length scales that cannot be resolved by the numerical grid, i.e. length scales smaller than

$$
\lambda_{\mathrm{g}}=2 \Delta,
$$

where $\Delta$ is the grid spacing. The factor 2 is due to the sampling theorem.

A precise definition for the subfilter scales is more problematic. Consider a large- $c_{S}$ LES. There is a length scale $\lambda_{f}$ at which the LES-generated three-dimensional TKE spectrum drops off significantly in comparison with the inertial-range $k^{-5 / 3}$-spectrum. If we define a decrease by a factor $e=2.7181 \ldots$ as significant and if we assume Pao's (1965) spectrum as a reasonable approximation for the postulated universal TKE spectrum of turbulence generated by a large- $c_{S}$ LES, $\lambda_{f}$ is approximately given by

$$
\exp \left(-\frac{3}{2} \alpha x_{f}^{4 / 3}\right)=\frac{1}{\mathrm{e}}
$$

where

$$
x_{f}=k_{f} \eta_{\mathrm{LES}}=\frac{2 \pi}{\lambda_{f}} \eta_{\mathrm{LES}} .
$$

We have shown that in the case of the Smagorinsky-type eddy-viscosity parameterization $\eta_{\text {LES }}$ is given by

$$
\eta_{\mathrm{LES}}=c_{S} \Delta
$$

and it follows that

$$
\lambda_{f}=2 \pi c_{S}\left(\frac{3}{2} \alpha\right)^{3 / 4} \Delta
$$

Inserting Lilly's value of the Smagorinsky coefficient,

$$
c_{S L}=\frac{1}{\pi}\left(\frac{2}{3} \alpha\right)^{3 / 4}
$$

see (3.13), leads to

$$
\lambda_{f}=2 \frac{c_{S}}{c_{S L}} \Delta=\frac{c_{S}}{c_{S L}} \lambda_{\mathrm{g}}
$$

Thus, subfilter scales may be considered length scales smaller than $\lambda_{f}$. Obviously, $\lambda_{f}$ equals $\lambda_{g}$ if Lilly's value for the Smagorinsky coefficient is used. In this case the terms subfilter and subgrid may be considered synomous. Using a 'non-optimal', i.e. a larger value of $c_{S}$, however, leads to $\lambda_{f}>\lambda_{g}$. In this case a length scale $\lambda$ larger than $\lambda_{g}$ but smaller than $\lambda_{f}$ can be numerically resolved but not physically. This is the case in a large- $c_{S}$ LES. Thus, it is necessary to distinguish carefully between 'physically resolved scales' and 'numerically resolved scales'.

\subsection{Non-homogeneous LES turbulence}

The main purpose of LES is to model turbulence under real conditions. Real turbulence is often inhomogeneous owing to rigid boundaries or to stable stratification. Thus, it is very important to establish the reliability of the LES technique for nonhomogeneous conditions. Recently, encouraging results of LES of a neutrally stratified boundary layer (Andren et al. 1994) and even of stably stratified flows (Kaltenbach et al. 1994; Schumann \& Gerz 1995) have been published.

A 'dynamic' subgrid model has been developed (Germano et al. 1991) that allows 
more of the information about the local and instantaneous state of the flow at the smallest resolved scales to be used in order to account for anisotropy at these scales. This procedure results in a locally and temporally changing Smagorinsky coefficient $c_{S}$. The utility of Germano et al.'s (1991) model has been called in question by Mason (1994). On the other hand, Kaltenbach et al. (1994, pp. 26 ff.) are in agreement with Germano et al.'s procedure: "Our simulations lack an inertial subrange which can hardly be obtained unless the resolution is much finer than we were able to provide. A common practice in LES of a wide variety of flows is to account for the lack of an inertial subrange by adjusting $c_{S G S}$ [ $c_{S}$ in the terminology of the present paper] in such a manner that energy is dissipated at a sufficient rate." 'Dynamic' models might be the only way to guarantee the applicability of the LES technique in the case of stably stratified turbulence if the turbulence is anisotropic at all resolved length scales. But such procedures are not quite satisfactory. Making use of them seems to be a step backwards from the original concept of LES since a major advantage of the LES technique lies in its conceptual simplicity.

Even worse than in the presence of stably stratified regions is the situation in the vicinity of rigid boundaries: "It is disappointing to find that the boundary regions in large-eddy simulations contain serious errors. This cannot, however, be considered too surprising, as close to the surface the potential rationality of the large-eddy simulation vanishes as the dominant eddy-scales become comparable with, and smaller than, the filter-scale" (Mason 1994, p. 17).

Generally a Smagorinsky-type LES is expected to be successful if the flow is fully turbulent, if the length $l_{S}$ is always significantly smaller than the local outer scale and larger than the local inner scale of the turbulence to be modelled, and if the turbulence to be modelled is statistically isotropic at length scales comparable with $l_{S}$. Since the outer scales are drastically reduced in regions with high static stability, realistic LES of flows under these conditions require a considerably smaller $l_{S}$ than, for example, an LES of a convective boundary layer.

Recently, subfilter models have been developed which take into account backscatter of subfilter energy into the resolved-scale regime and reduce the intrinsic determinism of the basic Smagorinsky model by introducing a stochastic force at the smallest resolvable scales (see, e.g., Mason \& Thomson 1992; Mason 1994; Schumann 1995). A further subfilter model is the structure function model put forward by Métais \& Lesieur (1992).

However, an interpretation of the relationships between the concepts developed in the present paper, which rely on classical models of homogeneous turbulence, and the more sophisticated subfilter closures mentioned above is not given here.

\subsection{Intermittency and Reynolds-number similarity}

Another problem of the LES technique is the effective Reynolds number of the LESgenerated turbulence. In LES the dissipation length $\eta$ of the real-world turbulence is replaced with $\eta_{\mathrm{LES}}=c_{S} \Delta$. Thus,

$$
R e_{\mathrm{LES}}=\left(\frac{\eta}{\eta_{\mathrm{LES}}}\right)^{4 / 3} R e,
$$

may be defined as the turbulent Reynolds number of LES-generated turbulence, where $R e$ is the Reynolds number of the real-world turbulence to be simulated by the LES, see $\S 2$. In the atmospheric boundary layer, $\eta$ is on the order of $1 \mathrm{~cm}$ but $\eta_{\text {LES }}$ is usually chosen on the order of $10 \mathrm{~m}$. In this case $\operatorname{Re}_{\mathrm{LES}}$ is about $10^{4}$ times smaller than the Reynolds number $R e$ of the turbulence to be modelled. One must be careful that 
$R e_{\text {LES }}$ remains sufficiently large. Otherwise the Reynolds number similarity might not be fulfilled, and the LES results could not be considered representative for a large-Re flow (see, e.g., Moeng \& Wyngaard 1988).

It is known, however, that some statistical properties of fully developed turbulence are not independent of the Reynolds number even at very large Re, i.e. even if the Reynolds number similarity is to be expected to be guaranteed. Wyngaard \& Tennekes (1970) observed that the skewness and kurtosis of the first derivative of the streamwise velocity component depend on $R e$. The 4/3-ratio between longitudinal and transversal velocity correlations that is expected from the classical theory (equation 24 in Kolmogorov 1941a) has been observed. See, e.g., Hauf (1984). Mestayer (1982), however, reported experiments that did not give support to the theoretical 4/3-ratio. Also the LES results by Moeng \& Wyngaard (1988) did not reproduce the theoretical value, compare also Schmidt \& Schumann (1989, p. 528). Mestayer suggested that the 4/3-ratio is to be expected mainly at the small-scale regime of the inertial subrange. This implies that the existence of a 4/3-ratio depends on the Reynolds number. Another Reynolds-number dependency has been suggested by Oncley et al. (1990). They found empirically a relation between von Kármán's constant $\kappa$ and the roughness Reynolds number, leading to a smaller $\kappa$ for smooth terrain than for rough terrain.

Moeng \& Wyngaard (1988) considered the influence of the spatial fluctuations of LES-generated energy dissipation $\varepsilon$ on the TKE budget and pointed out that the LES-generated Kolmogorov coefficient depends on the width of the $\varepsilon$ distribution. It seems that the small-scale intermittency of LES-generated turbulence is an important effect at least for simulations of the convective boundary layer.

Recently, Schumann (1995) investigated LES-generated turbulence for three different values of $c_{S}: 0.083,0.165$ and 0.330 . While $c_{S}=0.165$ (which corresponds to Lilly's $c_{S}$ for $\alpha=1.6$ ) led to a realistic inertial-range spectrum with $\alpha_{\text {LES }} \approx 1.6$ (see Erratum to Schumann 1995) the doubled and halved $c_{S}$ provided unrealistic spectra (Schumann 1995, p. 311). This appears to be incompatible with the presumption that the LES results are asymptotically independent of $c_{S}$ if $c_{S}$ is chosen larger than Lilly's $c_{S}$-value (see figure 2). Here I suggest a possible explanation of Schumann's (1995) finding. Let $N$ be the number of the grid points in one dimension. For simplicity we identify the side length $L$ of a cubic modelled volume with the outer scale of the LES-generated turbulence. Then we have

$$
N=\frac{L}{\Delta}=\frac{L}{l_{S}} \frac{l_{S}}{\Delta}=\frac{L}{\eta_{\mathrm{LES}}} \frac{l_{S}}{\Delta}=R e_{\mathrm{LES}}^{3 / 4} c_{S}
$$

and finally

$$
R e_{\text {LES }}=\left(\frac{N}{c_{S}}\right)^{4 / 3} .
$$

Thus, $R e_{\mathrm{LES}}$ is reduced if $c_{S}$ is enlarged and vice versa, provided that $N$ is kept constant. The number of grid points in Schumann's (1995) LES was $N=64$, corresponding to $R e_{\text {LES }}=7069(2823,1122)$ for $c_{S}=0.083(0.165,0.330)$. These values for $R e_{\text {LES }}$ are not very large, and a violation of the Reynolds-number similarity seems possible. It is to be expected that there is a critical $R e_{\text {LES }}$ and that the Smagorinsky fluid is not fully turbulent if $R e_{\text {LES }}$ is smaller than this critical value. In this case, LES cannot generate an inertial-range spectrum. Possibly, $R e_{\text {LES }}=1122$ is below this critical value. It would be of great interest to test the $c_{S}$-sensitivity of a basic 


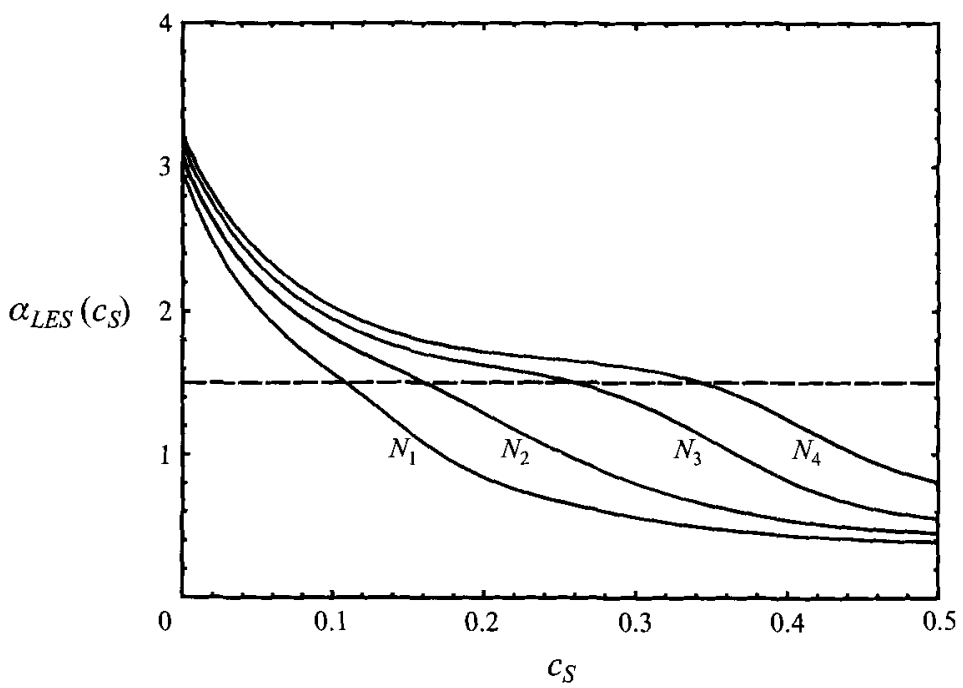

FIGURE 3. Qualitative picture of the expected behaviour of $\alpha_{\text {LES }}\left(c_{S}\right)$ for finite LES Reynolds numbers $R e_{\text {LES }}$, i.e. for finite numbers of grid points $N$, where $N_{1}<N_{2}<N_{3}<\ldots$. Note the expected plateau for large grids and the similarity to Lilly's (1967) $\alpha_{\mathrm{LES}}\left(c_{S}\right)$ model for small grids.

Smagorinsky-type LES without changing simultaneously the value of $R e_{\mathrm{LES}}$. This can be done by varying $N$ proportionally to $c_{S}$; in other words $\Delta$ has to be varied while $L$ and $l_{S}$ have to be kept constant.

Figure 3 shows qualitatively $\alpha_{\mathrm{LES}}\left(c_{S}\right)$ which is to be expected for different values of $N$ where $N_{1}<N_{2}<N_{3}<\ldots$. For a given $N$ it is to be expected that $\alpha_{\text {LES }}\left(c_{S}\right)$ drops off beyond a critical $c_{S}^{c}$ which is related to the critical LES Reynolds number $R e_{\mathrm{LES}}^{c}$ (which is expected to be not universal but to depend on the type of the flow) as follows:

$$
c_{S}^{c}=N \cdot\left(\operatorname{Re} e_{\mathrm{LES}}^{c}\right)^{-3 / 4} .
$$

It is to be expected that for small $N$ there is no plateau between Lilly's $c_{S}$ (the critical $c_{S}$ for discretization errors) and $c_{S}^{c}$ (the critical $c_{S}$ associated with the critical Reynolds number of the LES fluid). Such a plateau, however, is to be expected for larger $N$. In present-day LES we have $N \approx 100$, and it is possible that the expected plateau of $\alpha_{\text {LES }}\left(c_{S}\right)$ cannot be observed with such small values of $N$. Note that $\alpha_{\text {LES }}\left(c_{S}\right)$ for a small $N$ (see figure 3) exhibits some similarity with Lilly's (1967) model for $\alpha_{\text {LES }}\left(c_{S}\right)$, see figure 2. This similarity, however, appears to be just a misleading coincidence.

\section{Summary and conclusions}

Mason and his co-workers (Mason \& Callen 1986; Mason 1994; Mason \& Brown 1994) have pointed out that the effective spatial filter of an LES is not defined by the 'conceptual' filter that is applied to the Navier-Stokes equations in order to get the (continuous) LES equations. Neither is the filter determined by the numerical grid, provided the grid spacing is sufficiently small in comparison with the width of the 'conceptual' filter. Rather, the effective spatial filter is defined by the closure for the subfilter turbulence. The filter shape associated with the widely used Smagorinsky-type subfilter model, however, is unknown (Mason 1994). Mason's philosophy contrasts somewhat with the traditional philosophy (Lilly 1967; Leonard 1974), which assumes 
that the subfilter closure is a priori determined by the conceptual filter and that the width of the conceptual filter is simultaneously the grid spacing.

In the present paper, the LES equations have been considered equations of motion of specific hypothetical turbulent non-Newtonian fluids, called LES fluids. The Smagorinsky-fluid is by definition the LES fluid that is specified by the Smagorinskytype subfilter closure. A Kolmogorov-type similarity theory has been suggested which leads for very large LES Reynolds numbers to the TKE spectrum

$$
F(k)=\alpha_{\mathrm{LES}}\left(c_{S}\right) \bar{\varepsilon}^{2 / 3} k^{-5 / 3} f_{\mathrm{LES}}\left(x, c_{S}\right)
$$

where $f_{\mathrm{LES}}\left(x, c_{S}\right)$ is a dimensionless function of the dimensionless wavenumber $x=k l_{S}$. Here, $c_{S}$ is the Smagorinsky coefficient and $l_{S}=c_{S} A$ is the Smagorinsky length which scales the magnitude of the variable viscosity $v_{\text {LES }} \Delta$ is the mesh width (or in the case of anisotropic grid a measure of the mesh width).

The effective spatial filter is specified by the dimensionless function $f_{\text {LES }}\left(x, c_{S}\right)$. From the Leonard (1974) point of view $f_{\mathrm{LES}}\left(k l_{S}, c_{S}\right)$ is $A^{2}(k)$ where $A(k)$ is the Fourier transform of the conceptual filter. The key hypothesis of the present paper, however, is that the asymptote of $f_{\mathrm{LES}}\left(k l_{S}, c_{S}\right)$ for large $c_{S}$ is physically the Smagorinsky-fluid counterpart of Kolmogorov's (1941a) damping-function $f(x)$ which describes the drop-off of the TKE spectrum at wavenumbers in the vicinity of $\eta^{-1}$. This hypothesis is encouraged by the physical equivalence of $l_{S}$ and Kolmogorov's dissipation length $\eta$, which is shown by using Lilly's (1967) approximations for the ensemble averages $\bar{\varepsilon}$ and $\overline{v_{\text {LES }}}$. One of the anonymous reviewers remarked that the pivotal issue in this paper is the assumption that for homogeneous LES-generated turbulence the eddy viscosity can be replaced with a constant viscosity. It is clear that this assumption is closely related to the hypothesis of the equivalence of $\lim _{c_{S} \rightarrow \infty}\left(f_{\mathrm{LES}}\left(k l_{S}, c_{S}\right)\right)$ and $f(k \eta)$. But the 'equivalence hypothesis' appears to be more general since it implies that Kolmogorov's (1941a) similarity does not hold only for turbulent Navier-Stokes fluids but also for a certain (but up to now not specified) class of turbulent nonNewtonian fluids. In this respect, the concept of LES fluids seems reasonable, and it might be useful for defining alternative subfilter closures.

If Mason is right in saying that for homogeneous turbulence LES provides similar results to DNS with a constant (properly defined) viscosity, what is the advantage of LES compared with DNS? Both LES and DNS provide spatial and temporal distributions of energy dissipation rates, variances, and fluxes. However, there is a decisive difference: while in a Navier-Stokes fluid the viscosity is the material parameter and the dissipation length is variable, in a Smagorinsky fluid the dissipation length is the material parameter and the viscosity is variable. Thus, replacing a turbulent Navier-Stokes fluid with an equivalent turbulent LES fluid allows simulation of turbulence at arbitrarily large Reynolds numbers by making optimal use of the limited wavenumber-space regime that can be represented by a finite numerical grid.

We have combined Lilly's (1967) cut-off approximation with two classical dissipation -range models (Heisenberg 1948; Pao 1965). The combined models predict an intrinsic overestimation of the LES-generated Kolmogorov coefficient for finite values of $c_{S}$. For $c_{S}=0.2$ we obtain $16 \%$ overestimation from Heisenberg's (1948) model and $43 \%$ from Pao's (1965) model. The predicted overestimation becomes negligible for values of $c_{S}$ beyond about 0.5 . In other words both models predict non-negligible finite-difference-approximation errors for $c_{S}$ smaller than about 0.5 . It has been shown that the requirement $c_{S}>0.5$ is equivalent to the requirement $L<2 \eta$ ( $L$ is the wire length of a hot-wire anemometer) which has been recommended by experimentalists. 
It has been pointed out that for a constant number of grid points $N$ an enlargement of $c_{S}$ implies a reduction of the LES Reynolds number $R e_{\text {LES }}$, and it is to be expected that for a finite $N$ there is a critical value for $c_{S}$ associated with a critical $R e_{\text {LES }}$. Thus, for any finite $N$ there are two critical Smagorinsky coefficients: first Lilly's (1967) $c_{S}$, indicating for which values of $c_{S}$ finite-difference-approximation errors are important, and second a critical $c_{S}$ beyond which the Reynolds similarity is violated. Reasonable LES results are to be expected if $c_{S}$ is chosen not smaller than the first and not larger than the second critical Smagorinsky coefficient.

There appears to be a lack of a systematic study of homogeneous turbulence generated by a basic Smagorinsky-type finite-difference LES for different values of $c_{S}$ (between, say, 0.2 and 1.0) without changing simultaneously the LES Reynolds number. Such numerical experiments could empirically provide the function $f_{\mathrm{LES}}\left(x, c_{S}\right)$ and the LES-generated Kolmogorov-coefficient $\alpha_{\mathrm{LES}}\left(c_{S}\right)$. While it is to be expected that $f_{\mathrm{LES}}\left(x, c_{S}\right)$ and $\alpha_{\mathrm{LES}}\left(c_{S}\right)$ are not universal for small and intermediate $c_{S}$ but rather depend on the specific finite-difference-approximation scheme, it would be of great interest to get insight into the asymptotic shape of the Smagorinsky filter, $\lim _{c_{S} \rightarrow \infty} f_{\text {LES }}\left(x, c_{S}\right)$, and to determine the asymptotic value $\lim _{c_{S} \rightarrow \infty} \alpha_{\text {LES }}\left(c_{S}\right)$, i.e. the Kolmogorov coefficient for continuous Smagorinsky turbulence.

I thank P. J. Mason and U. Schumann for their valuable comments on earlier versions of this paper. Thanks also to R. Blender, P. Chilson, S. Raasch, V. Schilling, and Z. Sorbjan for helpful discussions and comments. I appreciate the valuable comments by three anonymous reviewers, and I would like to give thanks to F. Herbert and F. Fiedler who invited me to seminar talks in Frankfurt and Karlsruhe, respectively, on the topic of this paper, giving me the occasion to discuss my results.

\section{REFERENCES}

Andren, A., Brown, A. R., Graf, J., Mason, P. J., Moeng, C.-H., Niewstadt, F. T. M. \& Schumann, U. 1994 Large-eddy simulation of a neutrally stratified boundary layer: a comparison of four computer codes. Q. J. R. Met. Soc. 120, 1457-1484.

Bardina, J., Ferziger, J. H. \& Reynolds, W. C. 1983 Improved turbulence models based on largeeddy simulation of homogeneous, incompressible, turbulent flows. Rep. TF-19. Thermosciences Division, Department of Mechanical Engineering, Stanford University, Stanford, California.

Batchelor, G. K. 1953 The Theory of Homogeneous Turbulence. Cambridge University Press.

Chasnov, J. R. 1991 Simulation of the Kolmogorov inertial subrange using an improved subgrid model. Phys. Fluids A 3, 188-200.

Chen, S., Doolen, G. D., Krainchnan, R. H. \& She, Z.-S. 1993 On statistical correlations between velocity increments and locally averaged dissipation in homogeneous turbulence. Phys. Fluids A 5, 458-463.

Corrsin, S. 1961 Turbulent flow. Am. Sci. 49, 300-324.

DeARDORFF, J. W. 1970 A numerical study of three-dimensional turbulent channel flow at large Reynolds numbers. J. Fluid Mech. 41, 453-480.

DeardorfF, J. W. 1980 Stratocumulus-capped mixed layers derived from a three-dimensional model. Boundary-Layer Met. 18, 495-527.

Galperin, B. \& OrszaG, S. A. (Eds.) 1993 Large Eddy Simulation of Complex Engineering and Geophysical Flows. Cambridge University Press.

Germano, M. Piomelli, U., Moin, P. \& Cabot, W. H. 1991 A dynamic subgrid-scale eddy viscosity model. Phys, Fluids A 3, 1760-1765.

GöRTLER, H. 1975 Dimensionsanalyse. Springer.

HaUf, T. 1984 Turbulenzmessungen mit dem Forschungsflugzeug Falcon. Met. Rdsch. 37, 163-176.

HeisenberG, W. 1948 Zur statistischen Theorie der Turbulenz. Z. Phys. 124, 628-657. 
Hunt, J. C. R., Phillips O. M. \& Williams, D. (eds.) 1991 Turbulence and stochastic processes: Kolmogorov's ideas 50 years on. Proc. R. Soc. Lond. A 434, 1-240.

Kaltenbach, H.-J., Gerz, T., \& Schumann, U. 1994 Large-eddy simulation of homogeneous turbulence and diffusion in stably stratified shear flow. J. Fluid Mech. 280, 1-40.

Kolmogorov, A. N. $1941 a$ The local structure of turbulence in incompressible viscous fluid for very large Reynolds numbers. Dokl. Akad. Nauk SSSR 30, 301-305. (Engl. Transl. Proc. R. Soc. Lond. A 434, 9-13, 1991.)

Kolmogorov, A. N. $1941 \mathrm{~b}$ Dissipation of energy in the locally isotropic turbulence. Dokl. Akad. Nauk SSSR 30, 16-18. (Engl. Transl. Proc. R. Soc. Lond. A 434, 15-17, 1991.)

Kolmogorov, A. N. 1962 A refinement of previous hypotheses concerning the local structure of turbulence in a viscous incompressible fluid at high Reynolds number. J. Fluid Mech. 13, $82-85$.

Kutznetsov, V. R., Praskovsky, A. A. \& Sabelnikov, V. A. 1992 Fine-scale turbulence structure of intermittent shear flows. J. Fluid Mech. 243, 599-622.

LeONARD, A. 1974 Energy cascade in large eddy simulations of turbulent fluid flows. Adv. Geophys. 18 A, 237-248.

LiLLy, D. K. 1967 The representation of small-scale turbulence in numerical simulation experiments. Proc. IBM Sci. Comput. Symp. Environm. Sci. (Yorktown Heights, Nov. 14th to 15th 1966), pp. 195-210. Thomas J. Watson Res. Center, Yorktown Heights, N.Y.

Mason, P. J. 1994 Large-eddy simulation: A critical review of the technique. Q. J. R. Met. Soc. 120, $1-26$.

Mason, P. J. \& Brown, A. R. 1994 The sensitivity of large-eddy simulations of turbulent shear flow to subgrid models. Boundary-Layer Met. 70, 133-150.

Mason, P. J. \& Callen, N. S. 1986 On the magnitude of the subgrid-scale eddy coefficient in large-eddy simulations of turbulent channel flow. J. Fluid Mech. 162, 439-462.

Mason, P. J. \& ThOmson, D. J. 1992 Stochastic backscatter in large-eddy simulations of boundary layers. J. Fluid Mech. 242, 51-78.

McСomb, W. D. 1990 The Physics of Fluid Turbulence. Clarendon Press.

MestaYer, P. 1982 Local isotropy and anisotropy in a high-Reynolds-number turbulent boundary layer. $J$. Fluid Mech. 125, 475-503.

MÉTajs, O. \& Lf.SieUR, M. 1992 Spectral large-eddy simulation of isotropic and stably stratified turbulence. J. Fluid Mech. 239, 157-194.

Moeng, C.-H. \& WyngaARD, J. C. 1988 Spectral analysis of large-eddy simulations in the convective boundary layer. J. Atmos. Sci. 45, 3573-3587.

Muschinski, A. \& Roth, R. 1993 A local interpretation of Heisenberg's transfer theory. Beitr. Phys. Atmos. 66, 335- 346.

Oncley, S. P., Businger, J. A., Itsweire, E. C., Friehe, C. A., LaRue, J. C. \& Chang, S. S. 1990 Surface layer profiles and turbulence measurements over uniform land under nearneutral conditions. Preprints: Ninth Symp. on Turbulent Diffusion 30 April-3 May, 1990, Riso, Roskilde, Denmark, pp. 237-240. Am. Met. Soc., Boston, MA.

PAO, Y.-H. 1965 Structure of turbulent velocity and scalar fields at large wavenumbers. Phys. Fluids 8, $1063-1075$.

REYNOLDS, W. C. 1990 The potential and limitations of direct and large eddy simulations. In Whither Turbulence? Turbulence at the Crossroads (ed. J. L. Lumley), pp. 313-343. Springer.

Saddoughi S. G. \& Veerevalli, S. V. 1994 Local isotropy in turbulent boundary layers at high Reynolds number. J. Fluid Mech. 268, 333-372.

Schmidt, H. \& Schumann, U. 1989 Coherent structure of the convective boundary layer derived from large-eddy simulations. J. Fluid Mech. 200, 511-562.

SchumanN, U. 1994 On relations between constants in homogeneous turbulence models and Heisenberg's spectral model. Beitr. Phys. Atmos. 67, 141-147.

Schumann, U. 1995 a Stochastic backscatter of turbulence energy and scalar variance by random subgrid-scale fluxes. Proc. R. Soc. Lond. A 451, 293-318, and Erratum Proc. R. Soc. Lond. A 451, 811 .

Schumann, U. \& Gerz, T. 1995 Turbulent mixing in stably stratified shear flows. J. Appl. Met. 34, 33- 48.

Scotri, A., Meneveau, C. \& Lilly, D. K. 1993 Generalized Smagorinsky model for anisotropic grids. Phys. Fluids A 5, 2306-2308. 
SMAGORINSKY, J. 1963 General circulation experiments with the primitive equations. I. The basic experiment. Mon. Wea. Rev. 91, 99-164.

Tennekes, H. \& Lumley, J. L. 1972 A First Course in Turbulence. The MIT Press.

WyNGaARD, J. C. \& TENNEKES, H. 1970 Measurements of the small-scale structure of turbulence at moderate Reynolds numbers. Phys. Fluids 13, 1962-1969.

YAGLOM, A.M. 1981 Laws of small-scale turbulence in atmosphere and ocean (in commeration of the 40th anniversary of the theory of locally isotropic turbulence). Izv. Atmos. Ocean. Phys. 17, 919-935.

YAGLOM, A. M. 1994 A. N. Kolmogorov as a fluid mechanician and founder of a school in turbulence research. Ann. Rev. Fluid Mech. 26, 1-22. 